\title{
An Approach for Increasing Drive-Mode Bandwidth of MEMS Vibratory Gyroscopes
}

\author{
Cenk Acar and Andrei M. Shkel, Associate Member, IEEE, Associate Member, ASME
}

\begin{abstract}
The limitations of the photolithography-based micromachining technologies defines the upper-bound on the performance and robustness of micromachined gyroscopes. Conventional gyroscope designs based on matching (or near-matching) the drive and sense modes are extremely sensitive to variations in oscillatory system parameters that shift the natural frequencies and introduce quadrature errors. Nonconventional design concepts have been reported that increase bandwidth to improve robustness, but with the expense of response gain reduction. This paper presents a new approach that may yield robust vibratory MEMS gyroscopes with better gain characteristics while retaining the wide bandwidth. The approach is based on utilizing multiple drive-mode oscillators with incrementally spaced resonance frequencies to achieve wide-bandwidth response in the drive-mode, leading to improved robustness to structural and thermal parameter fluctuations. Enhanced mode-decoupling is achieved by distributing the linear drive-mode oscillators radially and symmetrically, to form a multidirectional linear drive-mode and a torsional sense-mode; minimizing quadrature error and zero-rate output. The approach has been implemented on bulk-micromachined prototypes fabricated in a silicon-on-insulator (SOI)-based process, and experimentally demonstrated.

[1285]
\end{abstract}

Index Terms-Inertial sensors, micromachined gyroscopes, MEMS, rate sensors.

\section{INTRODUCTION}

$\mathbf{T}$ HE tolerancing capabilities of the current photolithography processes and microfabrication techniques are inadequate compared to the requirements for production of high-performance inertial sensors. The resulting inherent imperfections in the mechanical structure significantly limits the performance, stability, and robustness of MEMS gyroscopes [3], [6]. Thus, fabrication and commercialization of high-performance and reliable MEMS gyroscopes that require picometer-scale displacement measurements of a vibratory mass have proven to be extremely challenging [1], [2].

The operation principle of the vast majority of all existing micromachined vibratory gyroscopes relies on the generation of a sinusoidal Coriolis force due to the combination of vibration of a proof-mass and an orthogonal angular-rate input. The proof mass is generally suspended above the substrate by a suspension system consisting of flexible beams. The overall dynamical system is typically a two degrees-of-freedom (2-DOF)

Manuscript received February 28, 2004; revised July 21, 2004. Subject Editor R. R. A. Syms.

The authors are with the University of California, Irvine, Mechanical and Aerospace Engineering Department, MicroSystems Laboratory EG2110, Irvine, CA 92697 USA (e-mail: cacar@uci.edu; ashkel@uci.edu; http://mems.eng.uci.edu).

Digital Object Identifier 10.1109/JMEMS.2005.844801 mass-spring-damper system, where the rotation-induced Coriolis force causes energy transfer to the sense-mode proportional to the angular rate input. In most of the reported micromachined vibratory rate gyroscopes, the proof mass is driven into resonance in the drive direction by an external sinusoidal electrostatic or electromagnetic force. When the gyroscope is subjected to an angular rotation, a sinusoidal Coriolis force is induced in the direction orthogonal to the drive-mode oscillation at the driving frequency. Ideally, it is desired to utilize resonance in both the drive and the sense modes, to attain the maximum possible response gain, and hence sensitivity. This is typically achieved by designing and electrostatically tuning the drive and sense resonant frequencies to match. Alternatively, the sensemode is designed to be slightly shifted from the drive-mode to improve robustness and thermal stability, while intentionally sacrificing gain and sensitivity [7].

The drive and sense mode matching (or near-matching) requirement in vibratory gyroscopes renders the system response very sensitive to variations in system parameters, e.g., due to fabrication imperfections and fluctuations in operating conditions, which shift the drive or sense resonant frequencies [6]. For the devices packaged in vacuum to enhance the sensitivity by increasing the drive and sense mode Q-factors, the bandwidths of the drive and sense frequency responses are extremely narrow; leading to much tighter mode-matching requirements and limited bandwidth of angular-rate detection. Extensive research has focused on design of symmetric drive and sense-mode suspensions for mode-matching and minimizing temperature dependence, [18]. However, especially for lightly-damped devices, it is recognized by many authors that the mode-matching requirement is well beyond fabrication tolerances; and none of the symmetric designs can provide the required degree of modematching without feedback control [4], [5].

Furthermore, extremely small imbalances in the gyroscope suspension due to fabrication imperfections introduce anisoelasticities, which result in undesired mode coupling often larger than the Coriolis motion. In order to suppress coupled oscillation and drift and to minimize the resulting zero-rate drift, various devices have been reported employing decoupled modes or independent suspension systems for the drive and sense modes [12]-[15]. The approach of structurally decoupling drive and sense modes led to the first integrated commercial MEMS gyroscopes mass-produced by Analog Devices [17].

The mode-matching problem and the quadrature error due to inherent fabrication imperfections are the two major challenges in MEMS gyroscope design. We have previously reported gyroscope systems that offer improved robustness by increasing the degree-of-freedom of the dynamical system [8], [9]. Even 


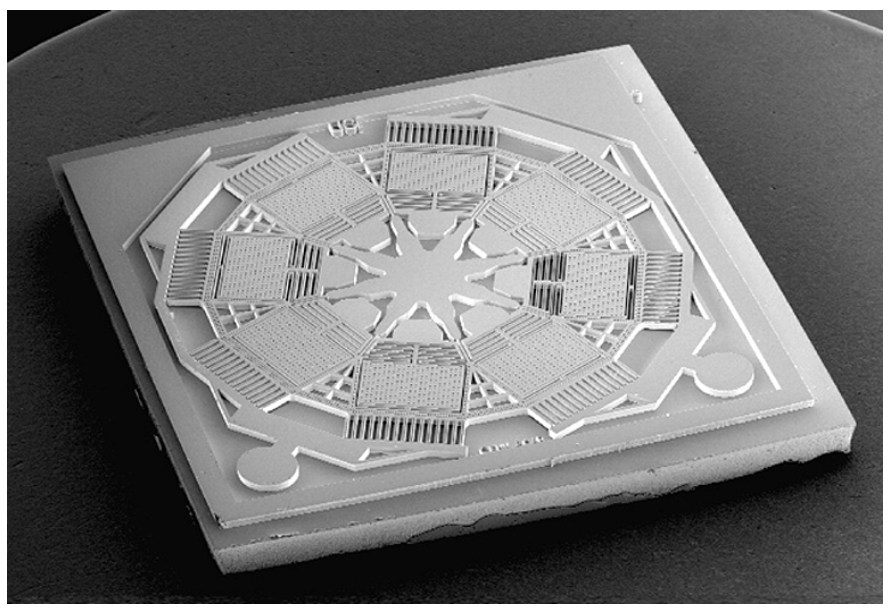

Fig. 1. Scanning electron microscope micrograph of a distributed-mass micromachined gyroscope prototype, utilizing multiple drive-mode oscillators with incrementally spaced resonance frequencies.

though increased-DOF gyroscope systems provide significantly increased bandwidth (over $1 \mathrm{kHz}$ ), this is achieved with the expense of sacrificing response gain. This paper presents a novel approach that may provide wider drive-mode bandwidth than conventional MEMS gyroscopes, with less sacrifice in response gain compared to previously reported wide-bandwidth devices. The concept based on utilizing multiple drive-mode oscillators with incrementally spaced resonance frequencies (see Fig. 1) was introduced in [10] by these authors, with the preliminary experimental results on the first generation prototypes presented in [11]. In this paper we generalize the approach in Section II, theoretically and experimentally explore the involved design tradeoffs to achieve a wide drive-mode bandwidth in Sections III and IV, and present the experimental characterization results that demonstrate the feasibility of the design concept in Section IV.

\section{THE APPROACH}

Since the Coriolis force, and the sense-mode response is directly proportional to the drive-mode oscillation amplitude, it is desired to enhance the drive-mode amplitude by increasing the $\mathrm{Q}$ factor with vacuum packaging and operating at the peak of the drive-mode resonance curve. However, large drive-mode amplitude and bandwidth cannot be achieved with a 1-DOF drive system at the same time. The proposed approach explores the possibility of increasing the drive-mode response bandwidth of micromachined gyroscopes, by utilizing multiple resonators with incrementally spaced resonant frequencies in the drivemode. The drive and sense modes are effectively decoupled by forming a multidirectional linear drive-mode that transmits the Coriolis force into a torsional sense-mode.

The design concept is based on forming multiple drive-mode oscillators, distributed symmetrically around the center of a supporting frame. The distributed drive-mode oscillators are driven in-phase toward the center of symmetry, and are structurally constrained in the tangential direction with respect to the supporting frame. Each oscillator is driven at the same drive frequency. In the presence of an angular rotation rate about the z-axis, a sinusoidal Coriolis force at the drive frequency is induced on each proof mass in the direction orthogonal to each drive-mode oscillation directions (see Fig. 2). Thus, each of the induced Coriolis force vectors lie in the tangential direction, combining to generate a resultant torque on the supporting frame. The net Coriolis torque excites the supporting frame into torsional oscillations about the $\mathrm{z}$-axis, which are detected by sense capacitors for angular rate measurement.

The multidirectional and axisymmetric nature of the drivemode oscillators offers several structural benefits over a conventional gyroscope design.

- Instability and drift due to mechanical coupling between the drive and sense modes is minimized, since the structure is designed to completely decouple the multidirectional linear drive-mode and the rotational sense-mode. Thus, zero-rate-output and quadrature error are significantly reduced in the presence of structural imperfections.

- $\quad$ The sensing electrodes are attached to the supporting frame, and do not respond to the drive-mode vibrations owing to the structural decoupling. This minimizes the noise in the response induced by the drive-mode oscillations.

- The torsional sense mode rejects external linear accelerations and vibrations.

- $\quad$ Since the drive forces applied to the drive-mode oscillators cancel out in all directions due to the radial symmetry, the net force on the structure is effectively suppressed. This results in near-zero reaction force induced on the anchor, thus minimizing energy emission to the substrate.

- The central single anchor structure minimizes the effects of packaging stresses and thermal gradients.

- The symmetry of the drive-mode oscillator structure about several axes also cancels the effects of directional residual stresses, and elastic anisotropy of the structural material.

\section{A. The Coriolis Response}

In the proposed approach, the distributed drive-mode oscillators are driven in-phase toward the center, and constrained in the tangential direction with respect to the supporting frame. The constrained dynamics of each proof-mass along the associated drive axis with respect to the supporting frame reduces to

$$
m_{i} \ddot{x}_{i}+c_{x} \dot{x}_{i}+k_{x} x_{i}=F_{d}
$$

where $m_{i}$ is the $i$ th proof-mass, and $x_{i}$ is the drive-mode response of the $i$ th mass. Thus, in the presence of an angular rotation rate about the z-axis, the Coriolis forces, which are proportional to drive direction oscillation amplitudes, induced on each proof mass are

$$
F_{c i}=2 m_{i} \Omega_{z} \dot{x}_{i} .
$$

The rotation-induced Coriolis forces are orthogonal to each of the drive-mode oscillation directions. Thus, each of the induced Coriolis force vectors lie in the tangential direction, combining 


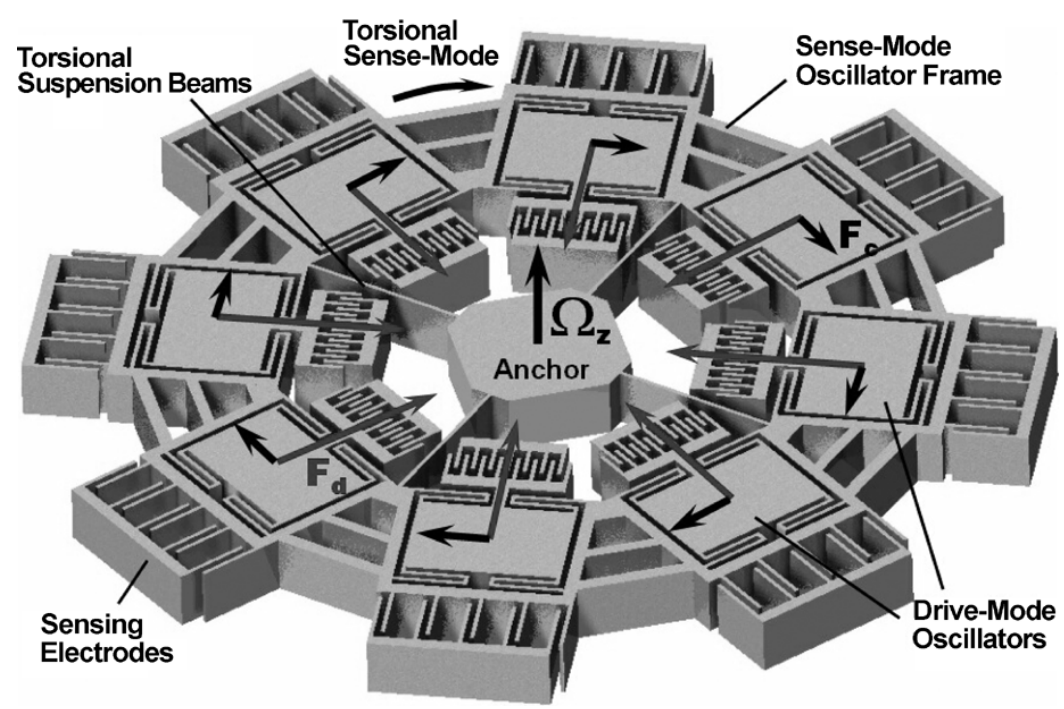

Fig. 2. Conceptual illustration of the distributed-mass gyroscope with eight symmetric drive-mode oscillators.

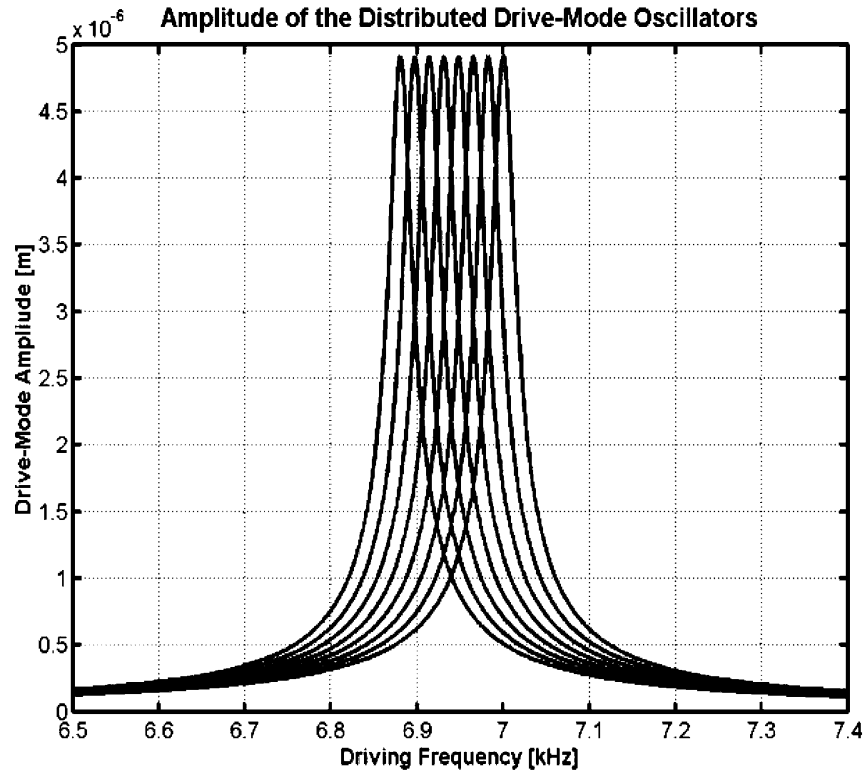

(a)

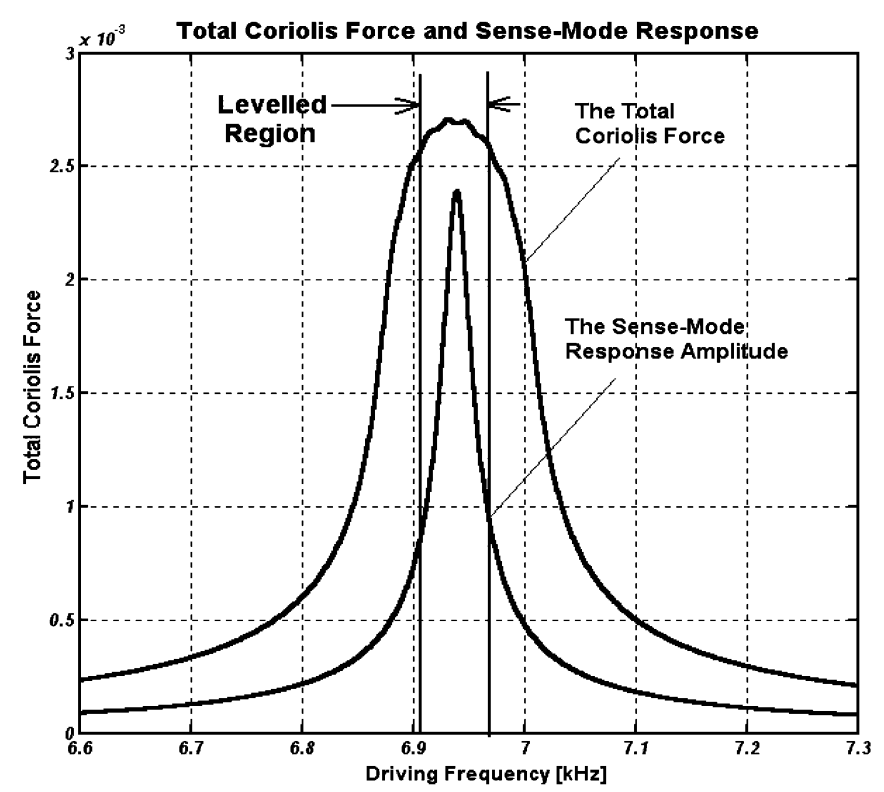

(b)

Fig. 3. (a) The frequency responses of the distributed drive-mode oscillators. (b) The frequency spectrum of the total Coriolis torque generated by the distributed drive-mode oscillators.

to form a resultant torque on the supporting frame. The net Coriolis torque generated as the combination of each Coriolis force becomes

$$
\vec{M}_{c}=\sum_{i=1}^{n} \vec{r}_{c} \times \vec{F}_{c i}=\sum_{i=1}^{n} 2 r_{c} m_{i} \Omega_{z} \dot{x}_{i} \cdot \widehat{k}
$$

where $\vec{r}_{c}$ is the position vector of the oscillator center-of-mass, and $\widehat{k}$ is the unit vector in the $z$-direction. The Coriolis torque $\vec{M}_{c}$ excites the supporting frame into torsional oscillations about the $\mathrm{z}$-axis, which is detected by the sense capacitors, providing measurement of angular rate. Assuming the rate input is constant and smaller compared to the driving frequency, the simplified equation of motion of the supporting frame in the sense-direction is

$$
I_{z} \ddot{\phi}+D_{z} \dot{\phi}+K_{z} \phi=M_{c}
$$

where $\phi$ is the torsional deflection of the supporting frame, $I_{z}$ denotes the moment of inertia of the supporting frame combined with the proof masses, $D_{z}$ is the sense-mode torsional damping ratio, and $K_{z}$ is the torsional stiffness of the suspension structure.

\section{B. Wide Bandwidth Operation for Improving Robustness}

In the presented design concept, a wide-bandwidth operation region is achieved in the drive-mode frequency response, by designing or actively tuning the resonance frequency of each drive-mode oscillator to be incrementally spaced [see Fig. 3(a)]. Since the tangential Coriolis forces induced on each proof mass jointly generate a resultant torque on the supporting frame, a "levelled" total Coriolis torque is achieved over a wide range of 


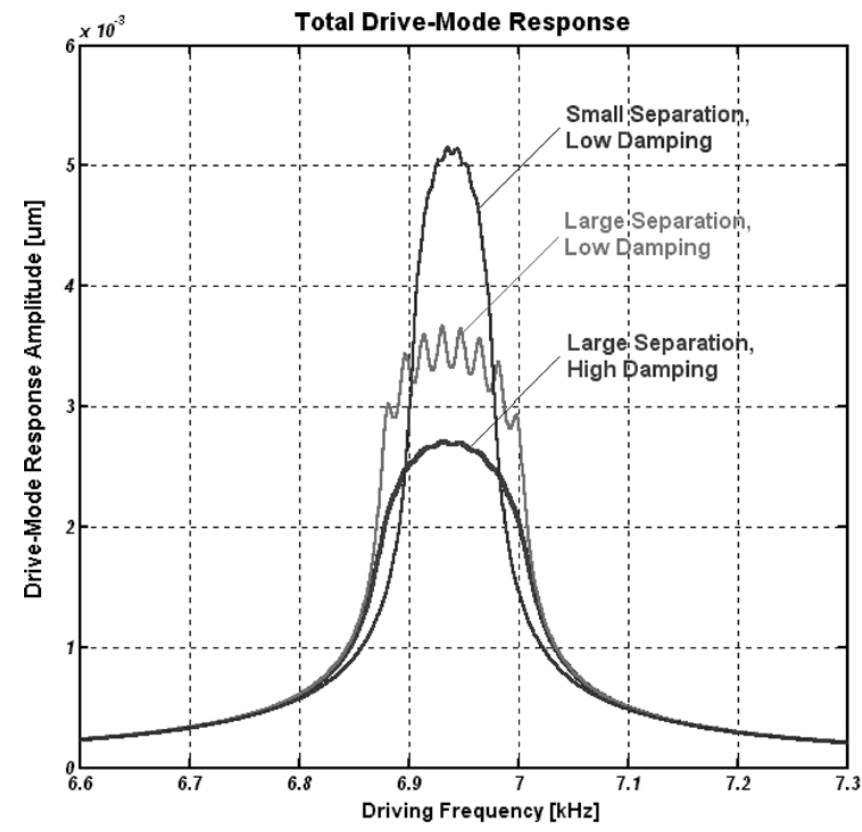

(a)

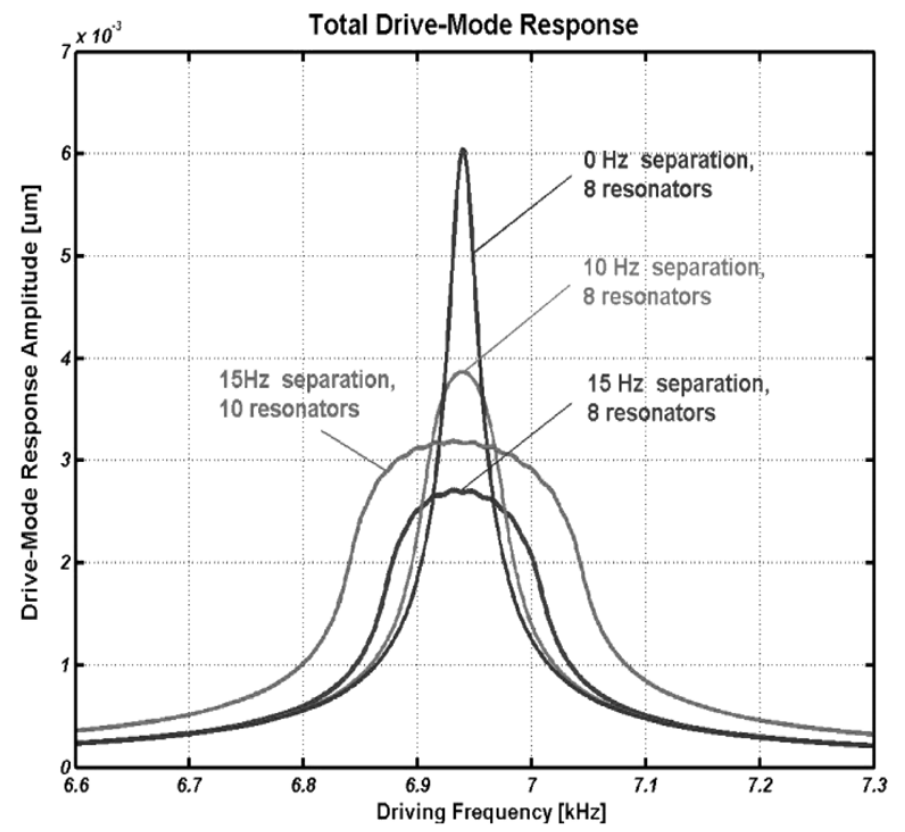

(b)

Fig. 4. (a) The effect of damping and resonance frequency separation on the drive-mode response. (b) The effect of frequency separation on the response gain and bandwidth (effecting sensitivity and robustness, respectively). The gain is maximized for zero frequency separation, and the overall bandwidth increases proportionally to spacing.

driving frequencies [see Fig. 3(b)]. The device is nominally operated in this levelled region of the Coriolis torque frequency response, so that fluctuations in system parameters that shift oscillator resonance frequencies will not result in a significant change in the total Coriolis torque. If the sense-mode resonance frequency is designed to be accommodated in the same frequency band [see Fig. 3(b)], the requirement on the degree of mode-matching is relaxed, and robustness against structural and thermal parameter fluctuations is achieved.

1) Driving Scheme: The drive-mode oscillators are driven at the same frequency inside the levelled frequency region. This assures that the sinusoidal Coriolis forces induced on each drivemode oscillator are at the same driving frequency. Thus, the sinusoidal Coriolis forces are superposed, and generate a resultant moment that excites the torsional sense-mode at the driving frequency.

During operation of the device, the forced oscillation amplitude of each oscillator will be different depending on the location of the drive frequency within the operation region, but the total drive-mode response will be constant at a known value. Thus, constant-amplitude control is not implemented on the oscillators, and the same signal is used to drive all of the oscillators for the purposes of demonstration of the design concept. In future implementations, a control architecture could be adapted that identifies the drive-mode parameters of each oscillator during calibration, and applies the appropriate drive signal to each oscillator so that the resonance amplitude of each is equal to a preset value.

2) Frequency Spacing Design: It should be noticed that the resonance frequency separation of the oscillators are dictated by the bandwidth of the response, and thus by damping. In order to obtain a levelled operation region in the drive-mode, the frequency separation should be less than the bandwidth of a single oscillator. If the separation of frequencies is large for low damping resonators, the resonance peaks become significant [see Fig. 4(a)], and the levelled operation region will not be achieved in the response. On the contrary, the total response will converge to a 1-DOF resonance peak as the frequency separation approaches zero, where the highest possible gain is attained with the narrowest bandwidth.

\section{THEORETICAL ANALYSIS OF THE TRADEOFFS}

The proposed design approach allows to widen the operation frequency range of the gyroscope drive-mode to achieve improved robustness, with the expense of sacrifice in the response amplitude. The optimal compromise between amplitude of the response and bandwidth (effecting sensitivity and robustness, respectively) can be obtained by selecting the frequency increments of the drive-mode oscillators.

As a numerical example, the response of a device consisting of eight drive-mode oscillators with resonance frequencies from 6.895 to $7 \mathrm{kHz}$ and a frequency spacing of $15 \mathrm{~Hz}$ will be considered. For $1^{0} / \mathrm{s}$ input angular rate and a $\mathrm{Q}$ factor of 100 in the drive and sense modes, the supporting frame of the distributedmass gyroscope will have an angular amplitude of response equal to $1.39 \times 10^{-6} \mathrm{rad}$, which is equivalent to $2.8 \times 10^{-3} \mu \mathrm{m}$ displacement at the sensing electrodes. If the frequency spacing of the drive-mode oscillators is decreased from $15 \mathrm{~Hz}$ to 10 $\mathrm{Hz}$, the amplitude of the response in the sense direction will increase from $2.8 \times 10^{-3} \mu \mathrm{m}$ to $3.9 \times 10^{-3} \mu \mathrm{m}$; while the response bandwidth will decrease from $200 \mathrm{~Hz}$ to $140 \mathrm{~Hz}$, which is still over an order of magnitude larger than the bandwidth of a single-mass conventional gyroscope. The bandwidth can be further widened by increasing the number of oscillators. In Fig. 4(b), the response of a gyroscope with 10 oscillators is 


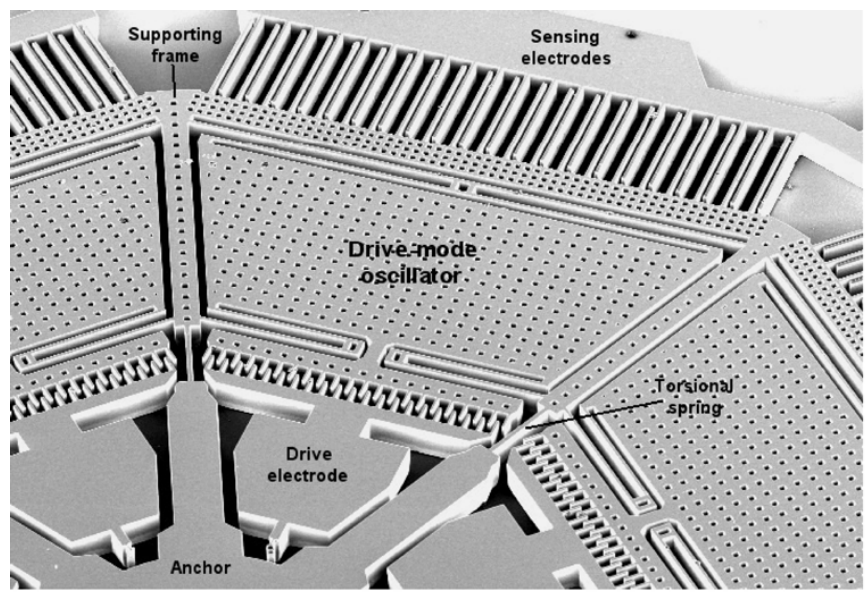

(a)

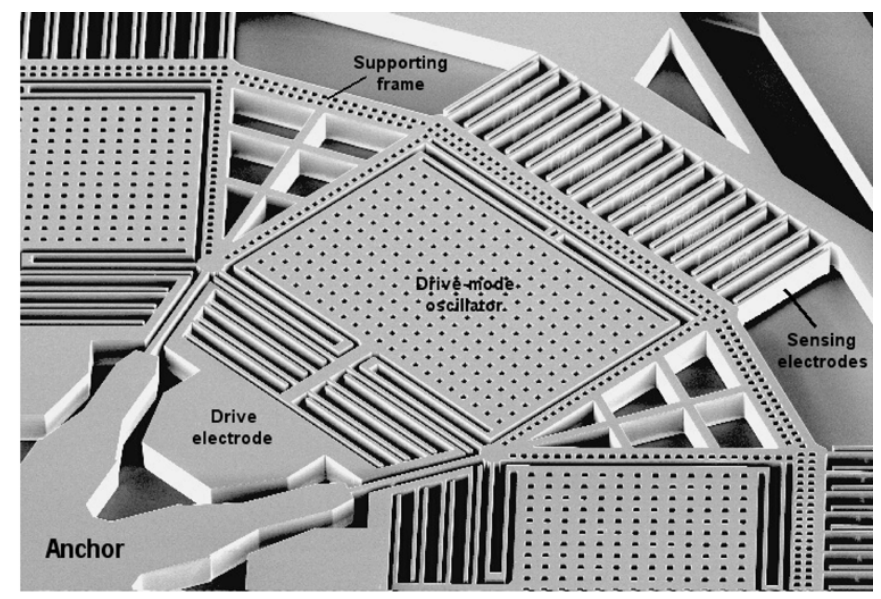

(b)

Fig. 5. SEM images of the characterized two different prototype structures: (a) the structure employing comb-drive actuation for large drive amplitudes and (b) the structure employing parallel-plate actuation for a wide electrostatic tuning range.

modeled along with 8-oscillator systems with 0,10 , and $15 \mathrm{~Hz}$ spacing; illustrating the effect of frequency separation and the number of oscillators. If the frequency separation is set to zero, the response gain will be at its maximum of $6.1 \times 10^{-3} \mu \mathrm{m}$, with a bandwidth of $100 \mathrm{~Hz}$. The tradeoffs between gain of the response (higher sensitivity) and the system bandwidth (increased robustness) are typically guided by application requirements.

\section{EXPERIMENTAL ANALYSIS OF THE TRADEOFFS}

\section{A. Fabrication of Prototypes}

The wide-bandwidth design concept was analyzed experimentally on the bulk-micromachined prototype structures, fabricated in the UCI Integrated Nano-Systems Research Facility (see Fig. 5). Two different prototype gyroscope structures utilizing the wide-bandwidth design concept were designed: one structure employing comb-drive actuation to achieve large drive amplitudes, and one structure employing parallel-plate actuation for a wide electrostatic tuning range.

For the fabrication of prototypes, a one-mask process based on SOI (Silicon-on-Insulator) wafers was developed and optimized for high-aspect ratio structures. The developed process relies on deep-reactive ion etching (DRIE) through the 100- $\mu \mathrm{m}$ -device layer, and front-side release of the structures by etching the Oxide layer in HF solution. The process and the device design was optimized to minimize notching at the oxide interface and excessive undercutting. The DRIE process was performed in an STS ICP, using $8 \mathrm{~s}$ etch step cycle with $130 \mathrm{sccm} \mathrm{SF}_{6}$ and $13 \mathrm{sccm} \mathrm{O}_{2}, 600 \mathrm{~W}$ coil power and $15 \mathrm{~W}$ platen power; and $5 \mathrm{~s}$ passivation step cycle time with $85 \mathrm{sccm}_{4} \mathrm{~F}_{6}, 600 \mathrm{~W}$ coil power, and $0 \mathrm{~W}$ platen power. In the device, $15 \mu \mathrm{m} \times 15 \mu \mathrm{m}$ holes were used to perforate the suspended structures, and 10 $\mu \mathrm{m}$ gaps were used in the sensing and actuation electrodes. The lowest etch rates were observed for the $15 \mu \mathrm{m} \times 15 \mu \mathrm{m}$ holes, at approximately $1.25 \mu \mathrm{m} / \mathrm{min}$, and $85 \mathrm{~min}$ DRIE time was used to assure complete through-etch while minimizing excessive undercutting in larger areas. The anchors were designed as unperforated areas larger than $40 \mu \mathrm{m} \times 40 \mu \mathrm{m}$ for 25 min release in $49 \% \mathrm{HF}$ solution. Each drive-mode oscillator was designed identically, although it will be shown in the next section that the natural frequency of each oscillator will be shifted due to fabrication imperfections. This phenomenon is exploited to naturally provide the required frequency spacing for this demonstration.

\section{B. Finite Element Analysis Results}

In order to optimize the system parameters and verify the validity of the theoretical analysis assumptions, the operational modes of the system were simulated using the Finite Element Analysis package MSC Nastran/Patran. Each drive-mode mass of the analyzed prototype system is $1240 \mu \mathrm{m} \times 770 \mu \mathrm{m}$, suspended by four $350 \mu \mathrm{m} \times 7 \mu \mathrm{m}$ folded springs; yielding a resonance frequency estimation of $7.15 \mathrm{kHz}$ with an elastic modulus of $130 \mathrm{GPa}$ for single-crystal Silicon in (100)-direction. Through FEA simulations, the resonance frequency of the drivemode oscillators were obtained at $6.98 \mathrm{kHz}$. The torsional sense mode resonance frequency of the structure about the sense axis was then located at $\omega_{z}=6.79 \mathrm{kHz}$ with four $296 \mu \mathrm{m} \times 10 \mu \mathrm{m}$ torsional suspension beams, by iteratively optimizing the beam length.

\section{Experimental Characterization Results}

The dynamic response of the linear drive-mode oscillators and the torsional sense-mode of the prototype gyroscope were characterized in an MMR Vacuum Probe Station. The frequency response of the prototype devices were acquired under varying pressure values and at room temperature, using off-chip transimpedance amplifiers with a feedback resistor of $R_{A}=1 \mathrm{M} \Omega$ connected to an HP Signal Analyzer in sine-sweep mode. The drive-mode frequency responses were acquired utilizing oneport actuation and detection, where a single electrode was used for both driving and sensing at the same time. The driving ac signal plus the dc bias voltage was imposed on the gyroscope structure through the anchor, and the actuation and detection port was directly connected to the transimpedance amplifier.

The resonance frequencies of the drive-mode resonators were observed to be scattered between $4.546 \mathrm{kHz}$ and $5.355 \mathrm{kHz}$ 

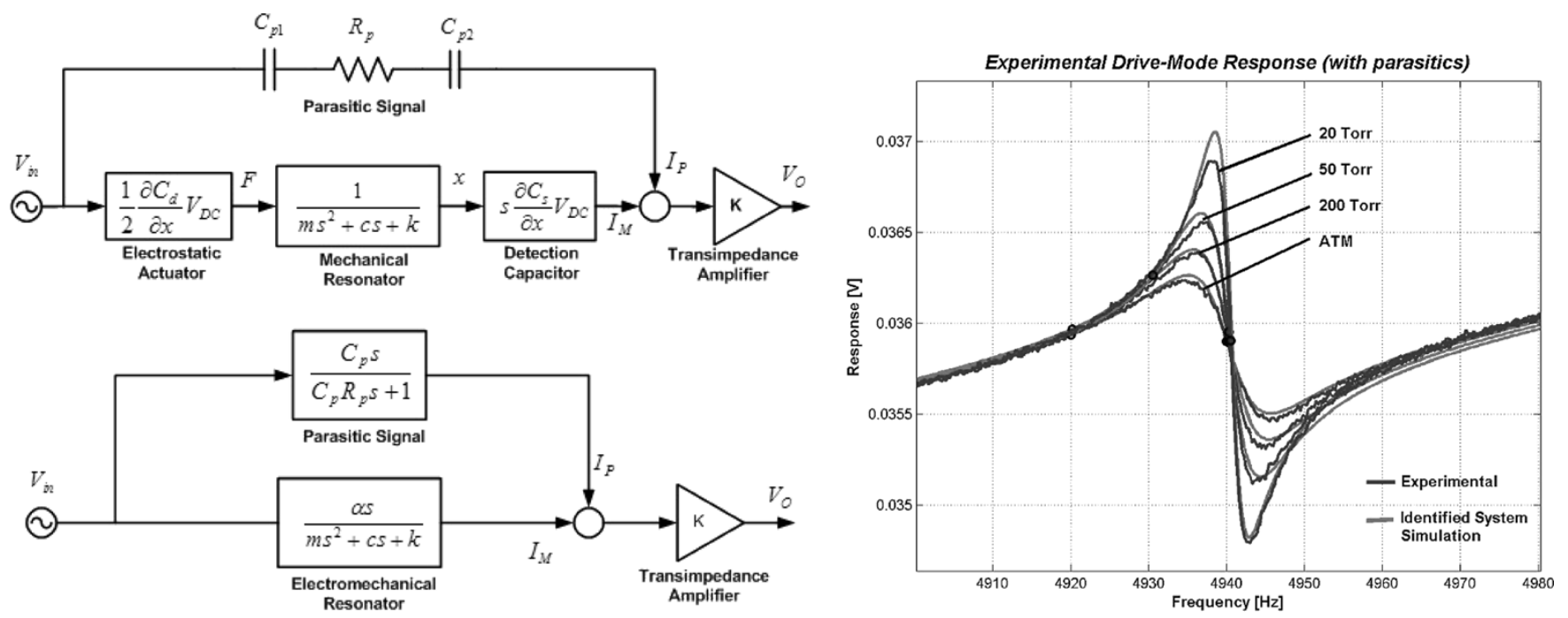

(a)

(b)

Fig. 6. (a) The transfer function model of the overall system, including the lumped parasitic capacitance, and the substrate resistance. (b) The measured frequency response at different pressure values, corrupted by the drive feed-through signal. The system parameters and the parasitics are effectively identified.

within a $809 \mathrm{~Hz}$ frequency band. The $16.36 \%$ maximum frequency deviation of the identically-designed drive-mode resonance frequencies results purely from the fabrication imperfections. The deviation of approximately $26 \%$ from the FEA results could be attributed to excessive lateral overetching during DRIE, the resolution of the mask used in fabrication, and the exposure and development steps of the photolithography process. In the presence of this wide-band scatter, measuring the bandwidth of the drive-mode oscillators is crucial to assess the feasibility of the design concept.

1) System Identification: The dynamical parameters of the drive-mode oscillators can be identified by electrostatically acquiring the frequency responses. However, the output signal is generally corrupted by the feed-through of the excitation signal to the detected signal over a lumped parasitic capacitance $C_{p}$ (e.g., between the bonding pads and the substrate, and between the drive and sense probes) and a finite substrate resistance $R_{p}$ in parallel to the ideal system dynamics [see Fig. 6(a)].

Even though this corrupted signal could be used to give a rough approximation of the system parameters, close estimation of parameters, even the resonance frequency, is not possible. Fortunately, the ideal system response can be extracted from the corrupted response with the analysis of the real and imaginary components of the response. The transfer function of the overall system, and the real and imaginary parts of the frequency response are

$$
\frac{\mathrm{V}_{\mathrm{o}}}{\mathrm{V}_{\mathrm{in}}}=K \frac{\alpha \mathrm{s}}{m \mathrm{~s}^{2}+c \mathrm{~s}+k}+K \frac{C_{p} \mathrm{~s}}{R_{p} C_{p} \mathrm{~s}+1}
$$

where

$$
\begin{aligned}
\operatorname{Re}\left(\frac{\mathrm{V}_{\mathrm{o}}}{\mathrm{V}_{\text {in }}}\right) & =\frac{K \alpha c \omega^{2}}{\left(k-m \omega^{2}\right)^{2}+c^{2} \omega^{2}}+\frac{K R_{p} C_{p}^{2} \omega^{2}}{\left(R_{p} C_{p} \omega\right)^{2}+1} \\
\text { and } & \operatorname{Im}\left(\frac{\mathrm{V}_{\mathrm{o}}}{\mathrm{V}_{\text {in }}}\right)=\frac{K \alpha\left(k-m \omega^{2}\right) \omega}{\left(k-m \omega^{2}\right)^{2}+c^{2} \omega^{2}}+\frac{K C_{p} \omega}{\left(R_{p} C_{p} \omega\right)^{2}+1}
\end{aligned}
$$

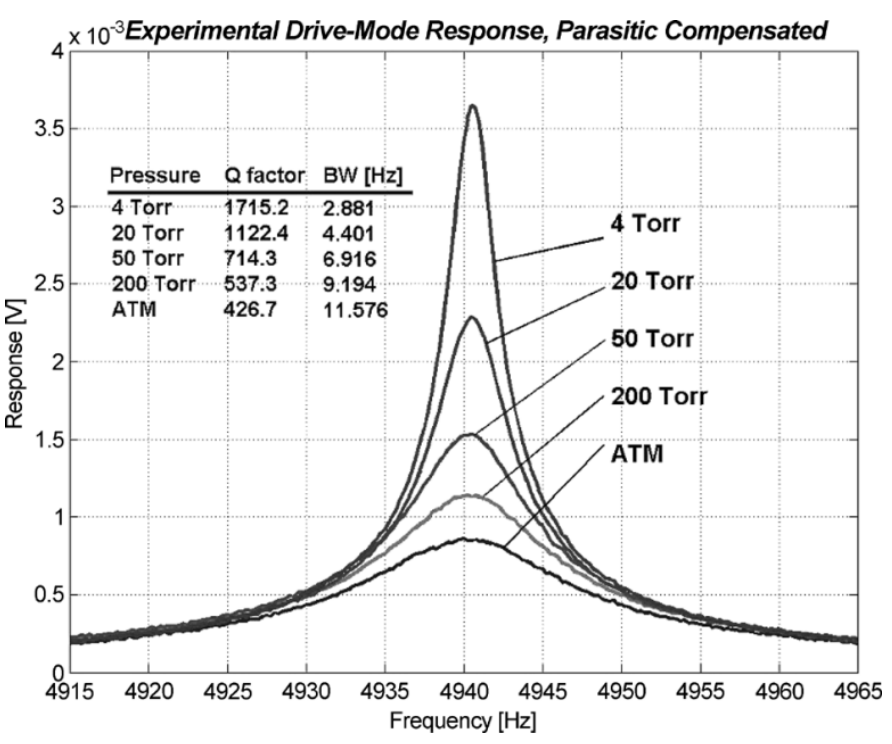

Fig. 7. Experimental measurements of the drive-mode frequency response of one of the oscillators, with numerical filtering of the parasitics. The clean output signal reflects the actual mechanical dynamics.

where $K$ is the transimpedance amplifier gain, and the constant $\alpha=(1 / 2)\left(\partial C_{\text {drive }} / \partial x\right) V_{\mathrm{dc}} \times\left(\partial C_{\text {sense }} / \partial x\right) V_{\mathrm{dc}}$ contains the coefficients for conversion of the input sine wave to the mechanical force, and the mechanical displacement to the motion induced current. The exact mechanical resonance point is very easily identified from real part of the response, since the real part reaches its maximum at the resonant frequency $\omega_{n}=\sqrt{k / m}$ regardless of the parasitics.

The real part of the response includes only the parasitic effects at the frequencies away from the resonance point, and the imaginary part of an ideal system's response is zero at the resonance point. Evaluating the real part at one frequency $\omega_{l}$ away from 


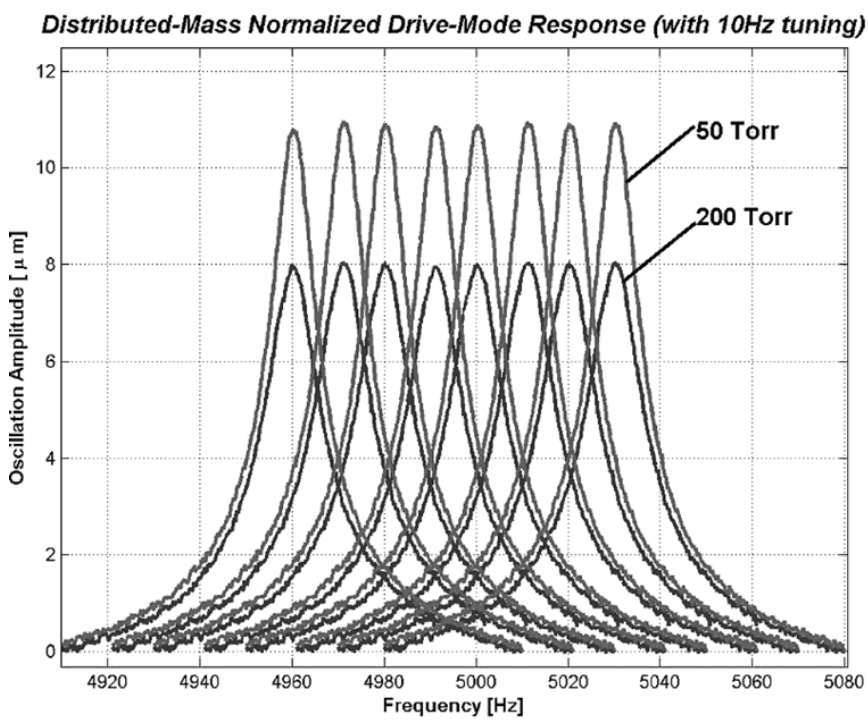

(a)

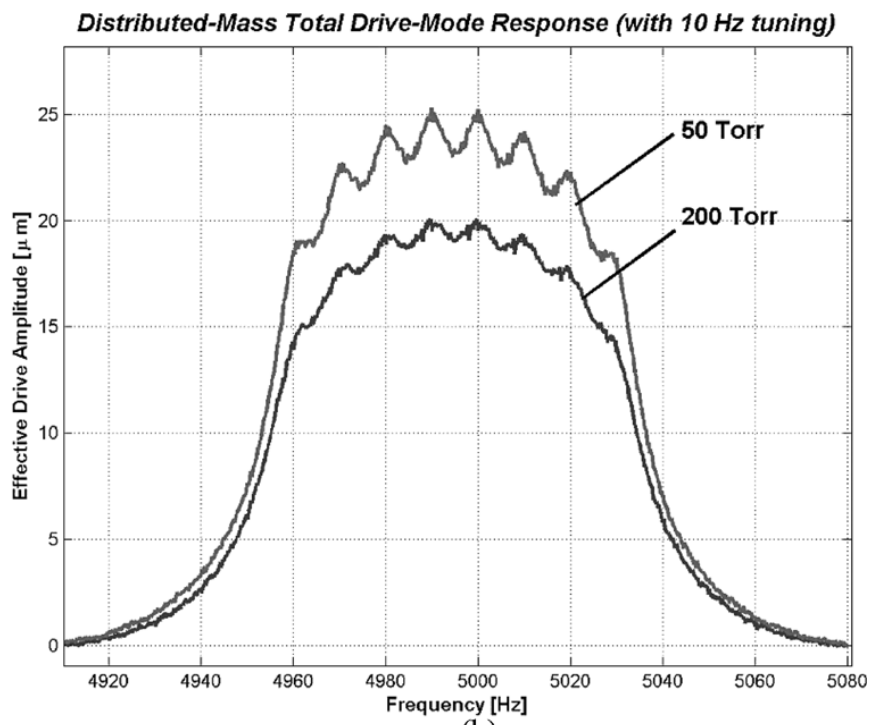

(b)

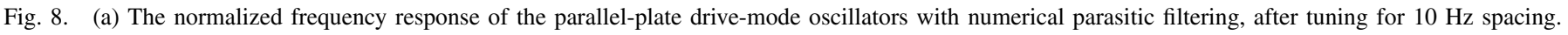

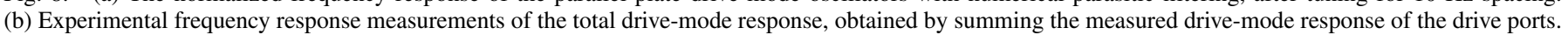

$\omega_{n}$, and the imaginary part at $\omega_{n}$ yields two nonlinear equation with two unknowns $R_{p}$ and $C_{p}$

$$
\begin{aligned}
\operatorname{Re}\left[\frac{\mathrm{V}_{\mathrm{o}}}{\mathrm{V}_{\mathrm{in}}}\left(\omega_{l}\right)\right] & =\frac{R_{p} C_{p}^{2} \omega_{l}^{2}}{\left(R_{p} C_{p} \omega_{l}\right)^{2}+1} \\
\operatorname{Im}\left[\frac{\mathrm{V}_{\mathrm{o}}}{\mathrm{V}_{\mathrm{in}}}\left(\omega_{n}\right)\right] & =\frac{C_{p} \omega_{n}}{\left(R_{p} C_{p} \omega_{n}\right)^{2}+1} .
\end{aligned}
$$

These two equations are solved simultaneously to identify the values of $R_{p}$ and $C_{p}$. Evaluating the real part at the resonance point, and the imaginary part of the response slightly away from resonance $\left(\omega_{m}\right)$ yields two equations for the two unknowns $\alpha$ and $c$

$$
\begin{aligned}
& \operatorname{Re}\left[\frac{\mathrm{V}_{\mathrm{o}}}{\mathrm{V}_{\mathrm{in}}}\left(\omega_{n}\right)\right]=\frac{\alpha}{c}+\frac{R_{p} C_{p}^{2} \omega_{n}^{2}}{\left(R_{p} C_{p} \omega_{n}\right)^{2}+1} \\
& \operatorname{Im}\left[\frac{\mathrm{V}_{\mathrm{o}}}{\mathrm{V}_{\mathrm{in}}}\left(\omega_{m}\right)\right]=\frac{\alpha\left(k-m \omega_{m}^{2}\right) \omega}{\left(k-m \omega_{m}^{2}\right)^{2}+c^{2} \omega_{m}^{2}}+\frac{C_{p} \omega_{m}}{\left(R_{p} C_{p} \omega_{m}\right)^{2}+1} .
\end{aligned}
$$

Solving these two equations simultaneously, the values of the electrical gain $\alpha$ and the damping coefficient $c$ are identified; yielding an accurate estimation of the $\mathrm{Q}$ factor and the bandwidth. Fig. 6(b) shows the experimentally acquired response, and the response of the identified model, verifying the estimation accuracy of the system parameters and parasitics. For the oscillator mass of $1.03 \times 10^{-7} \mathrm{~kg}$, the identified parameters using the proposed algorithm are $C_{p}=1.157 \mathrm{pF}, R_{p}=$ $1.701 \mathrm{M} \Omega$, and $\alpha=6.65 \times 10^{15}(\mathrm{VF} / \mathrm{m})^{2}$.

More importantly, having identified the parasitic terms in the real and imaginary parts of the response, these terms can be numerically filtered from the measured signal to reflect the actual mechanical dynamics, by subtracting the evaluated parasitic term at each frequency from the acquired trace. Fig. 7 presents the experimentally acquired frequency responses from atmospheric pressure to 4 Torr with numerical parasitic filtering, and the estimated $\mathrm{Q}$ factor and the bandwidth values.

2) Uniform Frequency Spacing With Tuning: The bandwidth of the drive-mode response even at atmospheric pressure

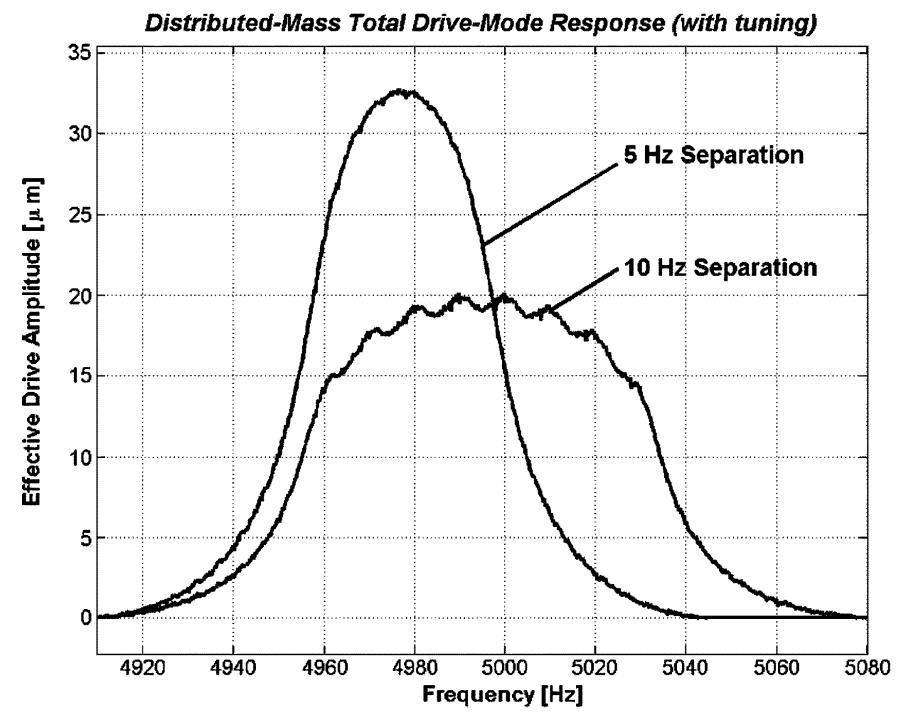

Fig. 9. The total drive-mode response measurements with $5 \mathrm{~Hz}$ spacing of the resonant frequencies, providing $65 \%$ larger gain, with the expense of less than $50 \mathrm{~Hz}$ bandwidth.

was observed to be too narrow to achieve wide-band operation without electrostatic tuning of the drive-mode frequencies. Thus, the prototype with the parallel-plate actuated drive-mode oscillators (see Fig. 1) which provides a wider range of electrostatic tuning was tested, and the resonance frequency of each oscillator was electrostatically tuned to achieve uniform and smaller frequency separation.

After electrostatic tuning of the parallel-plate oscillators for $10 \mathrm{~Hz}$ spacing [see Fig. 8(a)], the close spacing of the drive-mode resonance frequencies allowed all of the resonators to be excited together, to jointly generate a resultant Coriolis torque. The total Coriolis torque, which is estimated by summing the experimentally measured response of the eight drive-ports, was observed to provide a levelled range of over $90 \mathrm{~Hz}$ [see Fig. 8(b)]. When the experiments were repeated at 


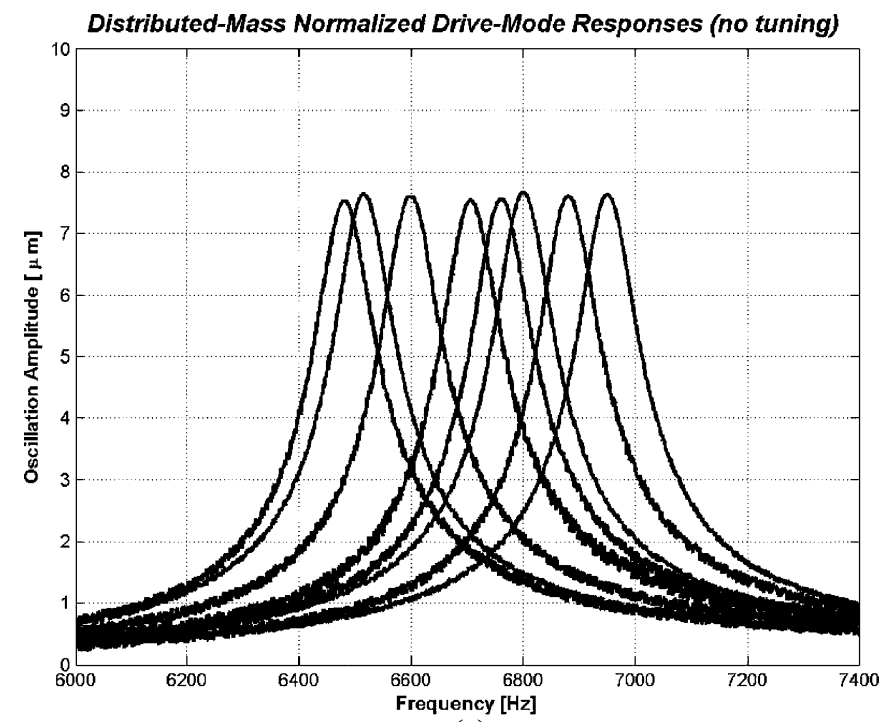

(a)

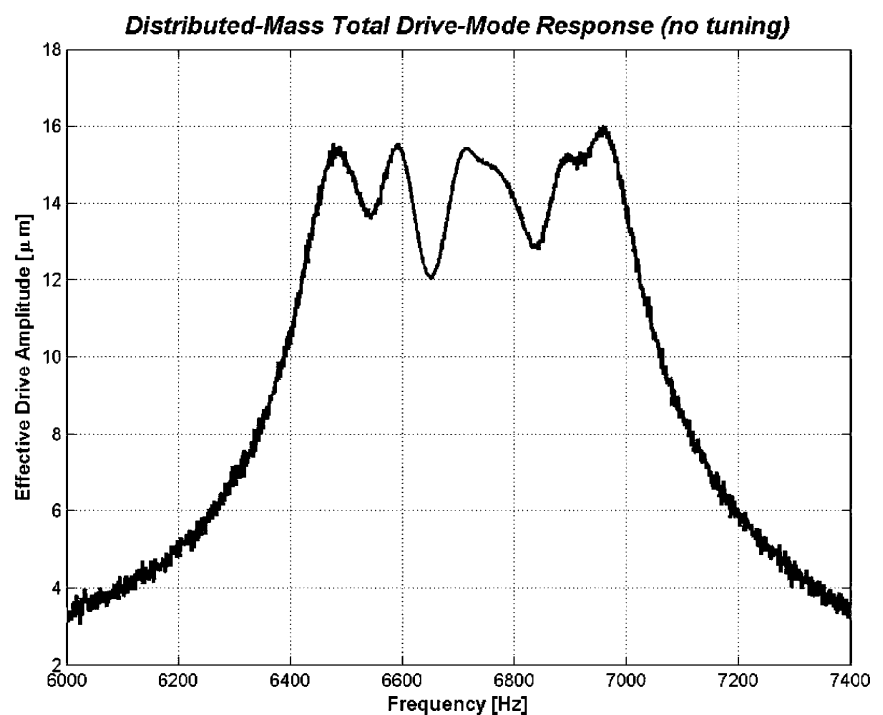

(b)

Fig. 10. (a) The natural frequency scatter of the drive-mode oscillators with 10- $\mu$ m-wide drive-mode beams. (b) Experimental frequency response measurements of the total drive-mode response at atmospheric pressure, with a maximum gain variation of $17.2 \%$ in the $600 \mathrm{~Hz}$ operating frequency region.

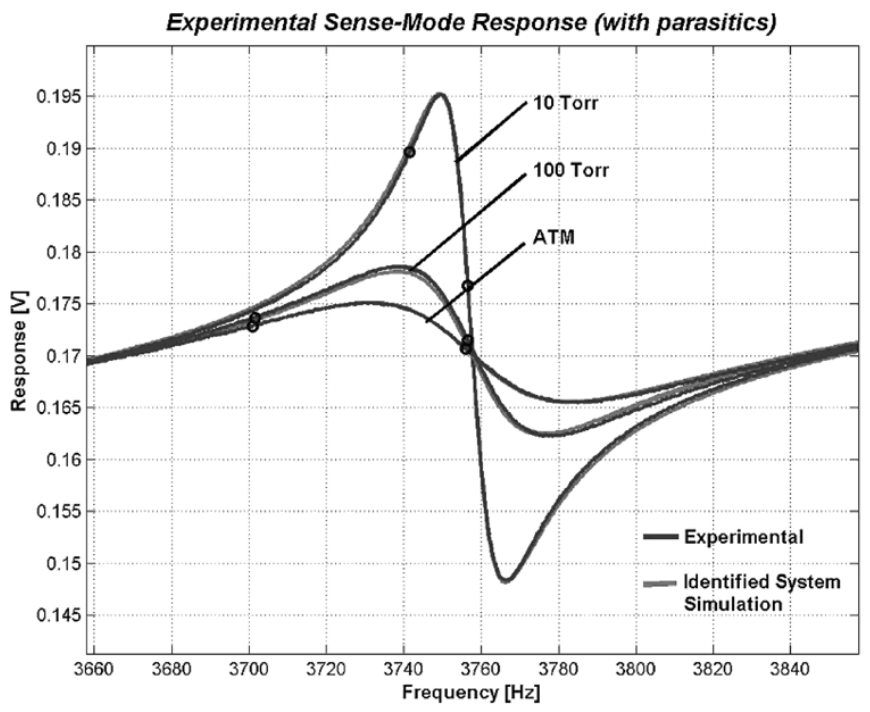

(a)

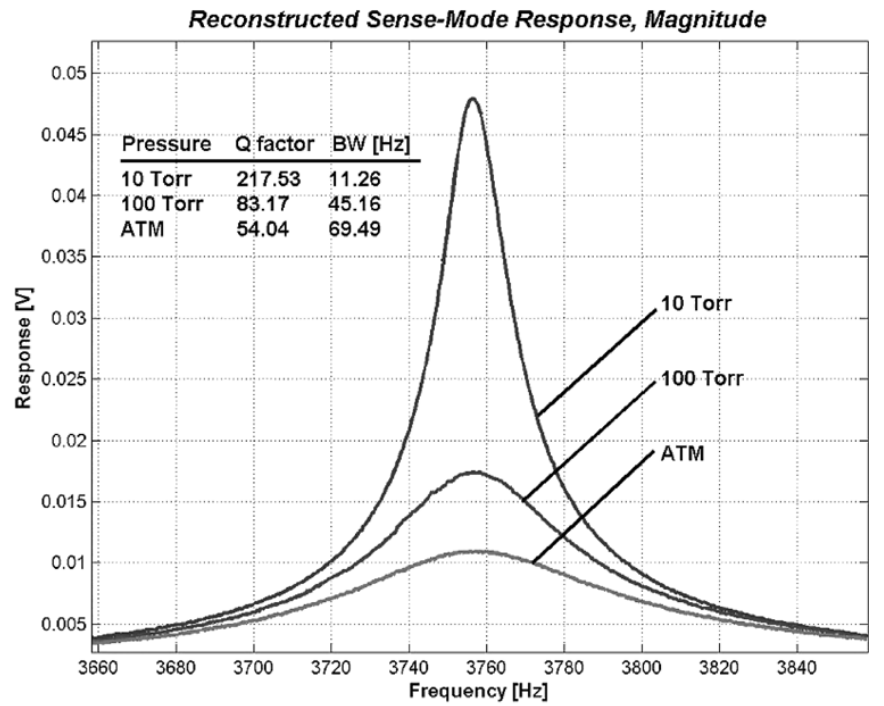

(b)

Fig. 11. (a) Experimental measurements of the torsional sense-mode frequency response, under different pressure conditions, with the parasitic feed-through. (b) The experimentally measured response amplitude with numerical filtering of the identified parasitics $C_{p}$ and $R_{p}$.

reduced pressures, the resonance peaks in the levelled region of the overall response became more emphasized, as was theoretically illustrated in the previous section. Based on the experimental results, it was concluded that 200 to 300 Torr is the optimal pressure for the parallel-plate devices to achieve a levelled wide-bandwidth drive-mode response with $10 \mathrm{~Hz}$ spacing. When the resonant frequencies were tuned for $5 \mathrm{~Hz}$ spacing, the total drive-mode response gain was measured to be $65 \%$ larger gain, but the bandwidth was observed to drop to $50 \mathrm{~Hz}$ (see Fig. 9).

3) Narrowband Frequency Spacing Without Tuning: In order to minimize the effects of suspension width variation due to fabrication imperfections on random scattering of the drive-mode resonance frequencies, a new generation of devices with $10 \mu \mathrm{m}$ wide drive-mode beams were designed, and fabricated using a higher resolution mask. The resonance frequencies of the drive-mode resonators with the wider suspension beams were observed to be scattered between 6.490 $\mathrm{kHz}$ and $6.920 \mathrm{kHz}$ in a $430 \mathrm{~Hz}$ frequency band, with a $6.21 \%$ maximum frequency deviation [see Fig. 10(a)]. The frequency separation of the resonators with $10 \mu \mathrm{m}$ wide beams was observed to provide over $600 \mathrm{~Hz}$ operating frequency region with levelled output in atmospheric pressure [see Fig. 10(b)]. However, the levelled region showed a maximum variation of $17.2 \%$ in the total response due to the nonuniform frequency separation.

4) Sense-Mode Characterization: The parasitics in the sense mode were modeled and identified similar to the drive mode analysis, yielding $C_{p}=7.18 \mathrm{pF}$ and $\alpha=5.34 \times 10^{13}(\mathrm{VF} / \mathrm{m})^{2}$. In Fig. 11(a), the experimentally acquired frequency responses of the torsional sense-mode and the identified system simulation are shown, verifying the 
estimation accuracy. Fig. 11(b) presents the experimentally acquired responses with numerical filtering of parasitics, from atmospheric pressure to 10 Torr, and the estimated Q factor and the bandwidth values. The sense-mode resonance frequency of the frame was measured at $3.758 \mathrm{kHz}$ with $20 \mathrm{~V}$ dc bias voltage.

\section{CONCLUSION}

A novel design approach based on utilizing multiple drive-mode oscillators with incrementally spaced resonance frequencies was presented, which provides wider drive-mode bandwidth in vibratory MEMS gyroscopes. The approach was theoretically illustrated, and experimentally verified. The linear drive-mode oscillators and the torsional sense-mode of the prototype gyroscope structures were characterized under varying pressure values. The resonance frequencies of the identically-designed drive-mode resonators were observed to be scattered within a $809 \mathrm{~Hz}$ frequency band, due to the fabrication imperfections. The bandwidth of the drive-mode response even at atmospheric pressure was observed to be too narrow to achieve wide-band operation. After electrostatic tuning of the parallel-plate oscillators for $10 \mathrm{~Hz}$ spacing, the close spacing of the drive-mode resonance frequencies allowed all of the resonators to be excited together, to jointly generate a resultant Coriolis torque with a levelled region of over 90 Hz. At pressures around 200 torr, the levelled wide-bandwidth drive-mode response was achieved together with sufficient off-resonance sense-mode gain, experimentally demonstrating the feasibility of the wide-bandwidth drive mode principle. The devices with $10 \mu \mathrm{m}$ wide suspension beams provided a levelled frequency region of $600 \mathrm{~Hz}$ with a maximum variation of $17.2 \%$ due to nonuniform spacing, demonstrating that the natural frequency scatter due to imperfections could be utilized to provide the required frequency spacing for wide bandwidth operation. Utilizing higher resolution fabrication technologies, the random scatter could be decreased further, and the oscillators could be ultimately designed with incrementally spaced resonant frequencies to provide the required uniform spacing.

\section{REFERENCES}

[1] N. Yazdi, F. Ayazi, and K. Najafi, "Micromachined inertial sensors," Proc. IEEE, vol. 86, no. 8, pp. 1640-1658, Aug. 1998.

[2] W. A. Clark, R. T. Howe, and R. Horowitz, "Surface micromachined Z-axis vibratory rate gyroscope," in Proc. Solid-State Sensors and Actuators, Hilton Head, SC, Jun. 1996.

[3] A. Shkel, R. Horowitz, A. Seshia, S. Park, and R. T. Howe, "Dynamics and control of micromachined gyroscopes," in Proc. American Control Conf., CA, 1999.

[4] S. Park and R. Horowitz, "Adaptive control for Z-axis MEMS gyroscopes," in Proc. American Control Conference, Arlington, VA, Jun. 2001.

[5] R. P. Leland, "Adaptive tuning for vibrational gyroscopes," in Proc. IEEE Conference on Decision and Control, Orlando, FL, Dec. 2001.

[6] A. Shkel, R. T. Howe, and R. Horowitz, "Modeling and simulation of micromachined gyroscopes in the presence of imperfections," in Proc. Int. Conf. on Modeling and Simulation of Microsystems, 1999.

[7] H. Xie and G. K. Fedder, "A DRIE CMOS-MEMS gyroscope," in IEEE Sensors 2002 Conference, Orlando, FL, Jun. 2002.

[8] C. Acar and A. Shkel, "Four degrees-of-freedom micromachined gyroscopes," J. Modeling Sim. Microsyst., vol. 2, pp. 71-82, 2001.

[9] _ - "Non-resonant micromachined gyroscopes with structural modedecoupling," IEEE Sensors J., vol. 3, no. 4, pp. 497-506, 2003.
[10] — - "Distributed-mass micromachined gyroscopes for enhanced mode-decoupling," in Proc. IEEE Sensors Conference, Toronto, Canada, Sep. 2003.

[11] — , "Enhancement of drive-mode bandwidth in MEMS vibratory gyroscopes utilizing multiple oscillators," in Proc. Solid-State Sensor and Actuator Workshop, Hilton Head Island, SC, Jun. 2004.

[12] J. A. Geen, "A path to low cost gyroscopy," in Solid-State Sensor and Actuator Workshop, Hilton-Head, 1998, pp. 51-54.

[13] W. Geiger et al., "Decoupled microgyros and the design principle DAVED," IEEE Sensors J., pp. 170-173, 2001.

[14] Y. Mochida et al., "A micromachined vibrating rate gyroscope with independent beams for drive and detection modes," Sens. Actuators A, Phys., vol. 80, pp. 170-178, 2000.

[15] M. Niu et al., "Design and characteristics of two-gimbals micro-gyroscopes fabricated with quasi-LIGA process," in Proc. Int. Conf. on Solid-State Sensor and Actuators, 1997, pp. 891-894.

[16] H. T. Lim, J. W. Song, J. G. Lee, and Y. K. Kim, “A few deg/hr resolvable low noise lateral microgyroscope," in Proc.IEEE MEMS Conference, NV, 2002, pp. 627-630.

[17] [Online]. Available: http://www.analogdevices.com

[18] Y. S. Hong, J. H. Lee, and S. H. Kim, "A laterally driven symmetric micro-resonator for gyroscopic applications," J. Micromech. Microeng., vol. 10, pp. 452-458, 2000.

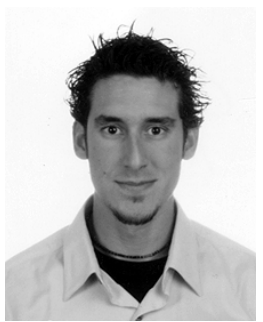

Cenk Acar was born in Turkey in 1977. He received the B.S. degree in mechanical engjneering from Bogazici University, Turkey, and the MS. and Ph.D. degrees in mechanical and aerospace engineering from University of California, Irvine.

His current research interests include design, modeling, fabrication, characterization, and control of microelectromechanical systems inertial sensors. He is the first author of over 20 publications on MEMS inertial sensors and currently has six pending patents.

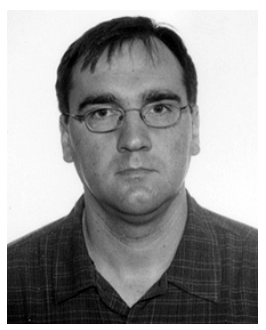

Andrei M. Shkel (A'04) received the Diploma (with excellence) in mechanics and mathematics from Lomonosov's Moscow State University, Russia, in 1991. In 1997, he received the Ph.D. degree in mechanical engineering from the University of Wisconsin-Madison.

$\mathrm{He}$ is on the faculty at the University of California-Irvine, where he is an Assistant Professor in the Department of Mechanical and Aerospace Engineering, in the Department of Electrical Engineering and Computer Sciences, and in the Department of Biomedical Engineering. He is also the Director of the UC1 Micro-Systems Laboratory. After receiving the Ph.D. degree, he joined Berkeley Sensor \& Actuator Center (BSAC) as a Postdoctoral Researcher. He then held research and consulting positions in several hi-tech and venture companies, including MEMSolutions, Inc., Solus Microtechnologies, Honeywell Corporation, Endevco, Inc., VIP Sensors, Silicon Valley Venture, etc. His professional interests, reflected in over 60 publications, include solid-state sensors and actuators, MEMS-based neuroprosthetics, sensor-based intelligence, and control theory. He holds three U.S. Patents (nine are pending) on micromachined angle-measuring gyroscope, design and fabrication of light manipulators and tunable optical filters, and hybrid surface micromachining processes.

Dr. Shkel has served on a number of editorial boards, including Guest Editor for two special issues of the IEEE SENSORS JOURNAL, General Chair of 2005 IEEE Sensors Conference, Editorial Board Member for the International Journal on Smart Structures and Systems, Vice General Chair and Publications Chair of 2002, 2003, and 2004 IEEE Sensors Conferences, Member of the Editorial Advisory Board (EAB) for the ISA magazine-SensorTech, and member of technical committees of 2001, 2002, 2003 SPIE, TMS 2003, and ACC 2001. He was awarded the 2002 George E. Brown, Jr. Award and was the recipient of 2001 Fellowship of the Japanese Advanced Science Institute. Dr. Shkel is an Associate Member of the American Society of Mechanical Engineers (ASME) and SPIE. 